\title{
Estertores tipo velcro
}

\author{
Velcro type rales
}

Elena Chevalier Chockee, MD; ${ }^{1}$ JaVier Leonardo Galindo, MD; ${ }^{2}$ Liliana Arias, MD; ${ }^{3}$
Jorge Luís Rodríguez, MD; ${ }^{4}$ Carlos Celis-Preciado, MD, MSc. ${ }^{5}$

En su libro De l'auscultation médiate ou Traité du diagnostic des maladies des poumons et du cœur, René Laennec definió y caracterizó a los crepitantes o estertores como sonidos cortos, discontinuos, explosivos y no musicales que predominan durante la inspiración, que se escuchan mejor en regiones pulmonares dependientes y durante respiraciones lentas y profundas; estos ruidos adventicios se producen por la apertura repentina de vías respiratorias pequeñas anormalmente cerradas (1-3).

Según la nomenclatura de la Sociedad Americana del Tórax (ATS), los estertores finos son de baja amplitud, de menor duración y de tono más alto que los estertores gruesos; semejan el sonido que se escucha al separar suavemente la tira de velcro en el manguito del tensiómetro, por lo que se denominaron estertores tipo velcro en 1970 por Dines y DeRemee (1-3).

Históricamente, los estertores finos o tipo velcro se han considerado representativos de la fibrosis pulmonar idiopática, en especial desde el estudio de Carrington y colaboradores en 1978 que analizó los datos de 272 pacientes con diversas enfermedades pulmonares intersticiales difusas documentadas por biopsia pulmonar (4). En este estudio los estertores tipo velcro se auscultaron en el $60 \%$ de los pacientes con neumonía intersticial, incluidos todos aquellos con diagnóstico histológico de neumonía intersticial usual (NIU), y solo en el $20 \%$ de los casos con sarcoidosis y otras enfermedades granulomatosas (4).

En 2012 Cottin y Cordier señalaron que la presencia de los estertores tipo velcro por auscultación podría ser la única aproximación desde el examen físico para lograr un diagnóstico temprano de la fibrosis pulmonar idiopática, ya que estos se auscultan de manera temprana en el curso de la enfermedad, primero en las áreas pulmonares basales, donde inicia el proceso patológico, con una progresión posterior a las zonas apicales (5).

Recientemente, 2 estudios reportaron la utilidad de los estertores tipo velcro en la evaluación del paciente con enfermedad pulmonar intersticial (6, 7). En el primero de ellos, Sellarés y colaboradores analizaron los datos de

${ }^{1}$ Fellow de Neumología, Universidad de Panamá, Hospital Santo Tomás. Ciudad de Panamá, Panamá.

${ }^{2}$ Neumólogo, Unidad de Neumología, Hospital Universitario Mayor. Bogotá D. C., Colombia.

${ }^{3}$ Radióloga, Departamento de Radiología e Imágenes Diagnósticas, Hospital Universitario San Ignacio. Profesora ad honorem Facultad de Medicina, Pontificia Universidad Javeriana. Bogotá D. C., Colombia.

${ }^{4}$ Patólogo, Departamento de Patología, Hospital Universitario San Ignacio. Profesor instructor, Facultad de Medicina, Pontificia Universidad Javeriana, Bogotá D. C., Colombia.

${ }^{5}$ Neumólogo, Unidad de Neumología, Departamento de Medicina Interna, Hospital Universitario San Ignacio. Profesor Asistente, Facultad de Medicina, Pontificia Universidad Javeriana. Bogotá D. C., Colombia.

Autor de correspondencia

Carlos Celis-Preciado. Correo electrónico: celisc@javeriana.edu.co.

Recibido: 8/9/2018. Aceptado: 9/12/2018. 
132 pacientes incluidos de forma prospectiva en un programa de enfermedad pulmonar intersticial de una institución de referencia en España; los sujetos se dividieron en 2 grupos de acuerdo con la presencia (63 $\%)$ o no $(37 \%)$ de estertores tipo velcro. El grupo con estertores tipo velcro presentó más tos y disnea al momento del diagnóstico, con valores de capacidad vital forzada $(p=0,002)$ y de difusión de monóxido de carbono $(p=0,04)$ más bajos, y con un índice pronóstico GAP más alto $(p=0,01)(6)$. Todos los pacientes con patrón de NIU en la tomografía axial computarizada de alta resolución (TACAR) del tórax y todos aquellos con diagnóstico final de fibrosis pulmonar idiopática presentaron estertores tipo velcro; en el análisis multivariado, la presencia de los estertores tipo velcro estuvo asociada de forma independiente con el patrón de NIU (6).

Por su parte, Sgalla y colaboradores investigaron la posible correlación de los estertores tipo velcro con la existencia de distintos patrones radiológicos de enfermedades pulmonares intersticiales fibróticas (7). Para ello, grabaron digitalmente la auscultación pulmonar de 148 pacientes previo a la realización de la TACAR. Al utilizar modelos multivariados de regresión, los datos mostraron que los estertores tipo velcro se asociaron con enfermedad pulmonar intersticial fibrótica en la TACAR (Odds ratio [OR]: 13,46; intervalo de confianza [IC] $95 \%$ : 5,85-30,96; $p<0,001)$ y más fuertemente con el patrón de NIU (OR: 19,8; IC 95 $\%: 5,28-74,25 ; p<0,001)$ (7). Así mismo, los estertores tipo velcro se asociaron de forma independiente con la extensión de ciertos hallazgos como la reticulación (OR: 2,04; IC $95 \%: 1,62-2,57 ; p<0,001$ ), la panalización (OR: 1,88; IC $95 \%$ : 1,24-2,83; $p<0,01$ ), el vidrio esmerilado (OR: 1,74; IC $95 \%$ : 1,29-2,32; $p<0,001$ ) y las bronquiectasias por tracción (OR: 1,55; IC $95 \%$ : $1,03-2,32 ; p<0,05)(7)$.
Los estertores tipo velcro también son un signo temprano de compromiso pulmonar en asbestosis y están presentes en la mayoría de los pacientes con neumonía intersticial no específica idiopática $(5,8)$. En general, la presencia de estos estertores junto con la disnea o una alteración del intercambio gaseoso pueden indicar enfermedad pulmonar intersticial aun ante la evidencia de una radiografía de tórax normal (9).

\section{Conflictos de interés}

Los autores no declaran conflictos de interés con el presente artículo.

\section{Referencias}

1. Pasterkamp H, Kraman SS, Wodicka GR. Respiratory sounds. Advances beyond the stethoscope. Am J Respir Crit Care Med. 1997;156(3 Pt 1):974-87.

2. Bohadana A, Izbicki G, Kraman SS. Fundamentals of lung auscultation. N Engl J Med. 2014;370(8):744-51.

3. Pasterkamp H, Brand PL, Everard M, Garcia-Marcos L, Melbye H, Priftis KN. Towards the standardisation of lung sound nomenclature. Eur Respir J. 2016;47(3):724-32.

4. Epler GR, Carrington CB, Gaensler EA. Crackles (rales) in the interstitial pulmonary diseases. Chest. 1978;73(3):333-9.

5. Cottin V, Cordier JF. Velcro crackles: the key for early diagnosis of idiopathic pulmonary fibrosis? Eur Respir J. 2012;40(3):519-21.

6. Sellarés J, Hernández-González F, Lucena CM, Paradela M, Brito-Zerón P, Prieto-González S, et al. Auscultation of velcro crackles is associated with usual interstitial pneumonia. Medicine (Baltimore). 2016;95(5):e2573.

7. Sgalla G, Walsh SLF, Sverzellati N, Fletcher S, Cerri S, Dimitrov B, et al. "Velcro-type" crackles predict specific radiologic features of fibrotic interstitial lung disease. BMC Pulm Med. 2018;18(1):103.

8. Flaherty KR, Martinez FJ. Nonspecific interstitial pneumonia. Semin Respir Crit Care Med. 2006;27(6):652-8.

9. Epler GR, McLoud TC, Gaensler EA, Mikus JP, Carrington CB. Normal chest roentgenograms in chronic diffuse infiltrative lung disease. N Engl J Med. 1978;298(17):934-9. 